\title{
AN EFFICIENT AERODYNAMIC SHAPE OPTIMIZATION FRAMEWORK FOR ROBUST DESIGN OF AIRFOILS USING SURROGATE MODELS
}

\author{
D. Maruyama, D. Liu, and S. Görtz \\ DLR, Institute of Aerodynamics and Flow Technology \\ Lilienthalplatz 7, 38108 Braunschweig, Germany \\ \{daigo.maruyama,dishi.liu,stefan.goertz\}@dlr.de
}

Keywords: Robust Design Optimization, Geometrical Uncertainties, Surrogate Model, Aerodynamic Shape Optimization, Computational Fluid Dynamics (CFD).

\begin{abstract}
This paper deals with developing an efficient Robust Design Optimization (RDO) framework. The goal is to obtain an aerodynamic shape that is less sensitive to small random geometry perturbations and to uncertain operational conditions. The initial shape is the RAE2822 airfoil which is parameterized with 10 design variables. The robust design formulation used is based on an expectation measure. The goal was to minimize the sum of the mean and standard deviation of the drag coefficient of the RAE 2822 airfoil for a given nominal lift coefficient. Here, we focus on improving the methods used for computing the statistics of the aerodynamic performance of the airfoil in every optimization cycle. A relatively small number of samples is evaluated with CFD and used to construct surrogate models based on Kriging and gradient-enhanced Kriging. The aerodynamic performance statistics, which are used to evaluate the robust objective function, are estimated by using quasi Monte Carlo (QMC) sampling with many samples evaluated on the surrogate models. A large number of geometrical uncertainties is parameterized by using a truncated Karhunen-Loève expansion, which enables a significant reduction of the dimensionality of the problem and thus of the surrogate models. By varying the number of samples used to build the surrogate model and by comparing the two types of surrogate modeling methods, it is confirmed that the robust objective function can be evaluated accurately with at most 30 CFD computations and corresponding adjoint computations.
\end{abstract}




\section{INTRODUCTION}

Computational Fluid Dynamics (CFD) has become a sophisticated tool and can be used for complex aerodynamic analysis and design of aircraft. Many traditional shape optimization problems have been solved by high fidelity CFD and the optimized configurations feature the desired aerodynamic performance under perfect conditions. However, the performance may degrade due to environmental and operational uncertainties, or manufacturing tolerances. Recently, robust design of aircraft under uncertainties is gaining interest in aerodynamics. In general, the high-fidelity CFD models used in deterministic shape optimization are expensive in terms of computational cost if applied to robust design.

In this paper, we are concerned with robust design optimization (RDO) of the transonic RAE2822 airfoil, which is a test case in the FP7 European project UMRIDA [1]. The functional of interest is the drag coefficient $\left(C_{d}\right)$. Random variations of the flight conditions such as angle of attack, $\alpha$, and Mach number, $M$, may occur during flight due to changing atmospheric conditions. The airfoil shape may also be subject to manufacturing tolerances and to degradation during operations. These factors are irreducible and considered as realistic uncertainties (aleatory uncertainties). On the other hand, numerical and modeling errors are an additional source of uncertainty that has an impact on the RDO results. These are reducible (epistemic) uncertainties. Figure 1 shows a simple depiction of these uncertainties in numerical flow simulations. Here, we focus on simulating the effect of irreducible uncertainties on the design, which are modeled using stochastic methods. We consider non-intrusive approaches, where we treat the flow solver as a black box and only use it to calculate the outputs that are required to evaluate the objective function. In the whole process, the flow solver settings, the mesh topology, and the geometry parameterization are carefully chosen and fixed. We consider the realistic uncertainties as input uncertainties with normal distributions when we evaluate the robustness of the design and we introduce our efficient RDO approach which combines a shape optimization framework with uncertainty quantification (UQ) methods.

There are two main issues that need to be considered when developing an RDO framework. One is how to evaluate stochastic values, such as the mean and standard deviation, accurately and efficiently. For example, in transonic flow, because of the strong non-linearity of the governing equations, the results from full Monte Carlo (MC) simulations and those derived from approximations by stochastic modeling could be different. The MC method is one of the most straightforward UQ methods, but comes at a much higher cost than other non-intrusive methods. The method of moments, polynomial chaos expansion and stochastic collocation methods are widely used non-intrusive approaches for evaluating stochastic values [2,3,4,5]. We propose a RDO framework based on low-discrepancy sampling methods combined with Kriging-based surrogate models to compute the stochastic values, which are used to evaluate the objective function to be optimized, accurately and efficiently. The other issue is how to deal with a large number of uncertain parameters. Here, a large number of geometrical uncertainties is parameterized by a Karhunen-Loève expansion to achieve a significant reduction of the number of uncertain inputs [6,7].

There are several measures of robustness [5] that can be used in RDO and reliability-based design optimization. In this paper, we limit ourselves to a robustness measure that is based on the sum of the mean and standard deviation of the drag coefficient as caused by the operational and geometrical uncertainties. Here we mainly discuss the accuracy of the robust objective function and how we can obtain the statistics efficiently. 


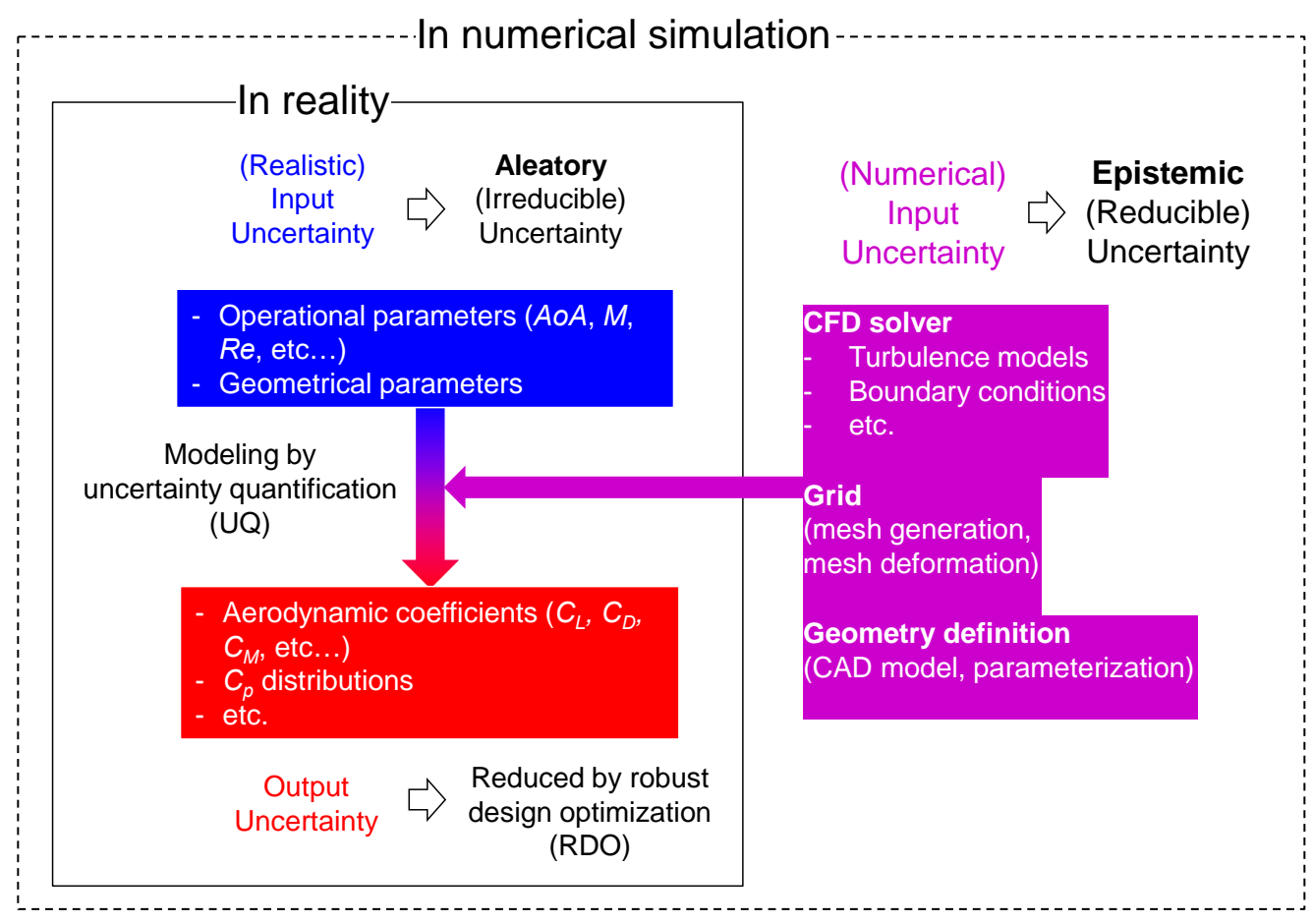

Figure 1: Overview of aleatory and epistemic uncertainties.

\section{UNCERTAINTY QUANTIFICATION FOR ROBUSTNESS MODELING}

\subsection{Description of test case and problem setting}

RDO is applied to the RAE2822 airfoil, which is one of the basic test cases in the UMRIDA project [1]. We consider the angle of attack, $\alpha$, the Mach number, $M$, and the geometry to be uncertain. The nominal condition of the angle of attack and Mach number are $\alpha=2.79$ deg. and $M=0.734$, respectively. The standard deviations of these operational parameters are $\alpha=0.1$ deg. and $M=0.005$ for the angle of attack and Mach number, respectively. These uncertainties are assumed to have a normal distribution. The Reynolds number is $\mathrm{Re}=6.5$ million and is not considered uncertain. The operational uncertainties are therefore expressed as $\alpha \sim N\left(\mu_{\alpha}, \sigma_{\alpha}\right)$ and $M \sim N\left(\mu_{M}, \sigma_{M}\right)$. The geometrical uncertainties are described in section 2.2 .

To evaluate the aerodynamic performance of the airfoil, DLR's flow solver TAU $[8,9,10]$ was used. Fully turbulent computations were performed with the negative Spalart-Allmaras turbulence model [11]. A quasi two-dimensional hybrid unstructured grid with prisms and tetrahedral elements was used for the RANS simulations. Figure 2 shows a detailed view of the grid around the RAE2822 airfoil, which is used as the baseline configuration in this paper. The number of grid nodes is approximately 29,000. During the RDO the geometry changes of the airfoil are realized by using a radial basis function (RBF) based mesh deformation tool developed at DLR [12]. In this paper, all 380 surface nodes are considered uncertain. This yields the issue of how to treat a large number of geometrical uncertainties. 


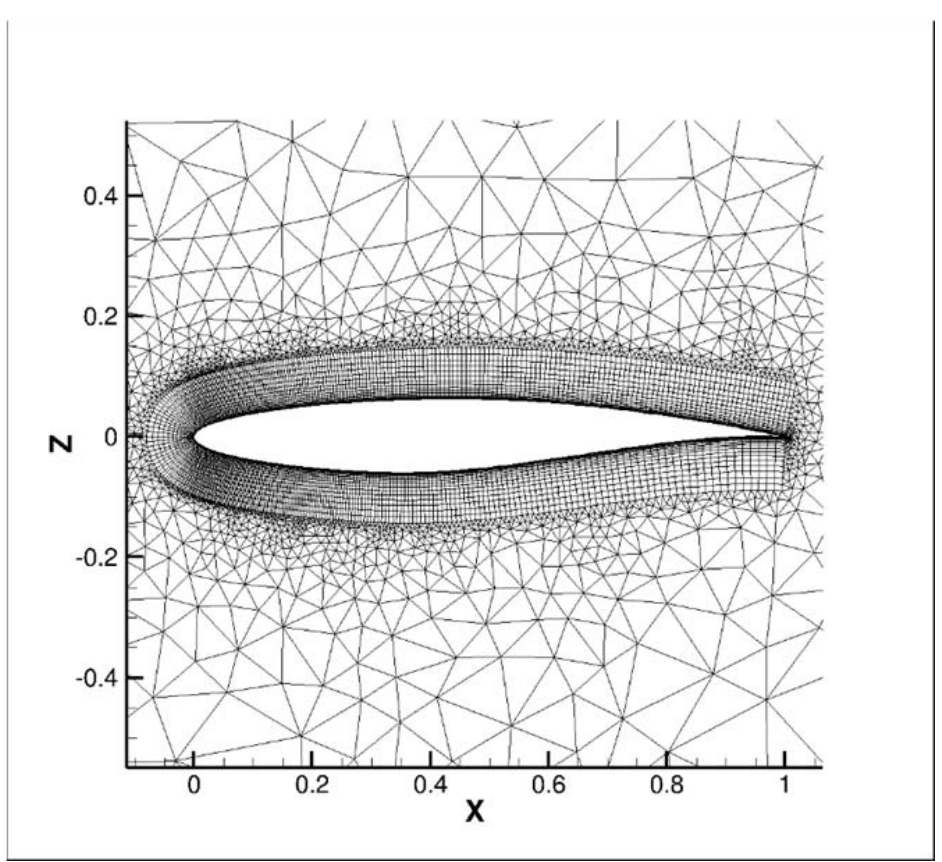

Figure 2: Detailed view of hybrid unstructured grid around RAE2822 airfoil.

\subsection{Modeling of a large number of geometrical uncertainties}

As briefly mentioned in the introduction, our approach to evaluate the cost function (the robust objective function) is based on a combination of design of experiment (DOE) methods and surrogate modeling. A reduction of the rather large number of geometrical uncertainties using a truncated Karhunen-Loève expansion (tKLE) [6,7] enables us to use much less samples in the DOE. We assume that random perturbations of the airfoil arise from manufacturing tolerances, for example, resulting in the surface to be smooth, i.e., the nodes on each surface are regarded to be correlated to each other.

The Karhunen-Loève expansion (KLE), which is also known as proper orthogonal decomposition (POD), is used to decompose correlated random fields into a linear combination of orthogonal functions, which are represented by uncorrelated random variables. In practice the orthogonal functions are carefully truncated to keep their number small. We assume a random perturbation $\theta(x)$ of each surface grid node in the direction normal to the airfoil surface on both the upper and lower surfaces with zero-mean normal distributions, i.e., $\theta(x) \sim N\left(0, \sigma_{\theta}(x)\right)$. Here, the standard deviation of the perturbation, $\sigma_{\theta}(x)$, is represented by the distribution represented in Fig. 3(i). This setting means that perturbations are largest at the mid-chord and zero at the leading and trailing edges. Thus, the maximum perturbation of the upper and lower surfaces is 0.0025 in total in this case, which is around half of the UMRIDA test case definition. Here, it is assumed that the random perturbations are spatially correlated by a Gaussian correlation function. Then, the KLE can decompose these correlated variables into uncorrelated ones. Only a relatively small number of the first several significant modes is retained. Finally, the correlated random perturbation $\theta(x)$ is transformed into a smaller number of independent standard Gaussian variables as $\xi \sim N(0,1)$, which are the newly modeled geometrical uncertainties. In our test case the number of random geometrical variables is reduced from 380 to 10 using the KLE. More details of this process can be found in [6]. Three 
realizations of the randomly perturbed geometry of the RAE2822 airfoil based on this parametrization are shown in Fig. 3(ii) as examples.

(i)

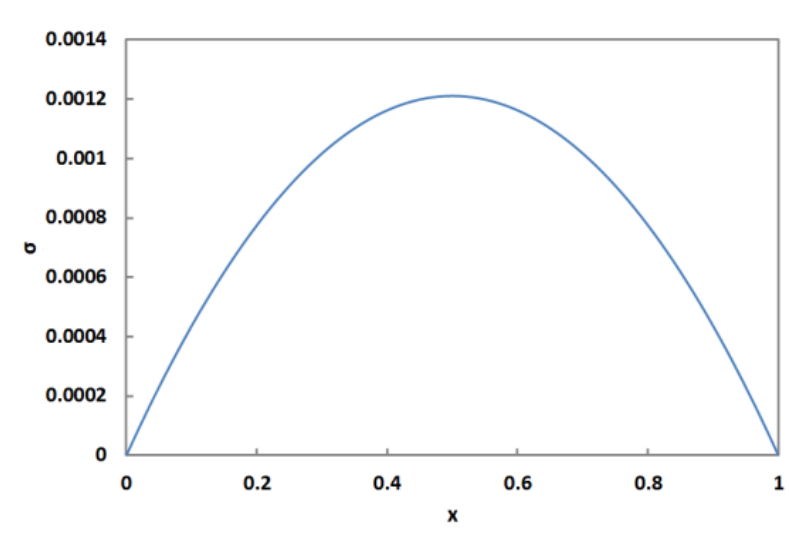

(ii)

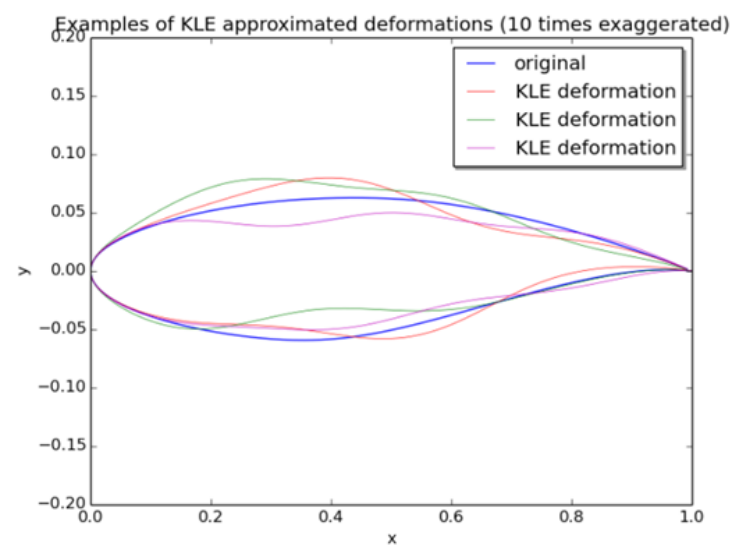

Figure 3: Distribution of the standard deviation of each surface node (left), and examples of the perturbed airfoil configurations of RAE2822 parameterized by truncated Karhunen-Loève expansion (tKLE) (right).

\subsection{Design of experiments and surrogate models}

The statistics, which are used to evaluate the robustness measure in every optimization cycle, are approximated using a combination of a DOE method and a surrogate model. The Sobol sequence based quasi Monte Carlo (QMC) $[13,14,15]$ was used as a DOE method to generate samples of the above uncertain input parameters in the domain of $\mu \pm 3 \sigma$. The samples are used to construct Kriging-based surrogate models of the functional of interest $[2,3,16]$. A further reduction of the computational cost is achieved by constructing gradient-assisted surrogate models, so called gradient-enhanced Kriging (GEK) [16], such that the gradient of the function of interest with respect to the uncertain input parameters are used as secondary DOE samples. Both Kriging and GEK are response surface methods used to statistically evaluate the function values belonging to an unsampled combination input parameters based on Bayesian inference. In both methods, maximum likelihood estimation (MLE) is adopted to optimize parameters of the correlation function. In this study, a Gaussian correlation function was used. Both Kriging and GEK as implemented in DLR's surrogate and reduced-order modeling toolbox SMARTy were used. The gradients with respect to the operational and geometrical parameters supplied to GEK were obtained by an adjoint method available in the DLR TAU-code [17]. The advantage of the adjoint method in this context is that all partial gradients of one cost function can be computed with a single adjoint solve.

Once a surrogate model is constructed, it can be used to evaluate the statistic of the functional of interest. The errors in the statistics computed with the help of the surrogate model can be made small by applying a MC method with a large number of samples such as $10^{6}$. For example, the mean and standard deviation can be evaluated with an error to the true value of around $0.01 \%$ by using the MC. Note that it is feasible to evaluate such a large number of samples using the surrogate model, while this is not feasible when using the CFD solver itself. 


\section{ROBUST DESIGN OPTIMIZATION FRAMEWORK}

The UQ approaches described in Section 2 are applied to evaluate the robust objective function. Once the objective function value is determined, existing deterministic optimization algorithms are applicable. Figure 4 shows the framework of the deterministic optimization part. We use an in-house aerodynamic shape optimization toolbox called Pyranha [18] as the basis in this part. As in deterministic optimization, the objective functions, the design variables, and the constraints can be introduced. In this case the constraints are a constant thickness-chord ratio $(t / c)$ and a constant lift coefficient $\left(C_{l}\right)$. Therefore, the parameterization to define the nominal shape is applied to the camber line. In this paper, we used Bernstein polynomials. The objective or cost function, $f$, is a measure of the robustness of the design:

$$
\left.\begin{array}{cc}
\min _{\chi} & f(\chi) \\
\text { s.t. } & t / c=\text { const. } \\
& C_{l}=\text { const. }
\end{array}\right\}
$$

where $\chi$ denotes the deterministic design variables. The design variables are the output of the ROD. The computation of the cost function at each optimization cycle is described later and depicted in Fig. 5.

Compared to deterministic design optimization (DDO), an additional target-lift CFD calculation is required before the cost function can be evaluated to determine the mean angle of attack $\mu_{\alpha}$ that satisfies the nominal $C_{l}$ constraint. Here, $C_{l}$ is fixed to 0.791 . The design variables, which are denoted by $\chi$, are represented by 10 Bernstein polynomials that are applied to the camber line. The Subplex algorithm [18] was used in this paper to optimize the cost function $f$ according to Eq. (1). It is based on the Simplex and Nelder-Mead algorithms. Different from the Simplex algorithm, the Subplex searches for the convex hull in several decomposed low dimensional subspaces. Thus, the Subplex algorithm is generally more efficient than the Simplex for many design variables. Moreover, it is applicable to noisy cost functions [19].

Figure 5 shows more details of the stochastic modeling part concerned with computing the cost function. In this paper, the cost function $f$ is the sum the mean, $\mu$, and the standard deviation, $\sigma$, of the drag coefficient $\left(C_{d}\right)$ :

$$
f(\chi, \mathbf{a}, \xi) \equiv \mu_{C d}(\chi, \mathbf{a}, \xi)+\sigma_{C d}(\chi, \mathbf{a}, \xi)
$$

where $\chi$, $\mathbf{a}$, and $\xi$ are the design variables, the operational uncertainties, and the geometrical uncertainties given by the tKLE parameters, respectively. The number of parameters $\chi, \mathbf{a}$, and $\xi$ are 10,2 , and 10 , respectively. Note that the design variables, $\chi$, are deterministic and describe the nominal airfoil geometry at each iteration of the optimization process.

As described in Section 2, $\mu_{C d}(\chi, \mathbf{a}, \xi)$ and $\sigma_{C d}(\chi, \mathbf{a}, \xi)$ are calculated by constructing a surrogate model of $C_{d}$ based on a limited number of CFD computations. This is detailed in the flowchart in Fig. 5. At first, QMC sampling with a limited number in the range $\mu \pm 3 \sigma$ for the uncertainty parameters a and $\xi$ is performed. This covers $99.97 \%$ of the cumulative density function (cdf) of the normally distributed input uncertainties. Here, the uncertainty parameters are angle of attack, Mach number and 10 geometrical uncertainties given by 10 tKLE parameters. Therefore, the dimension is 12. In this research, the number of QMC samples was set to 12 or 30 , respectively. 


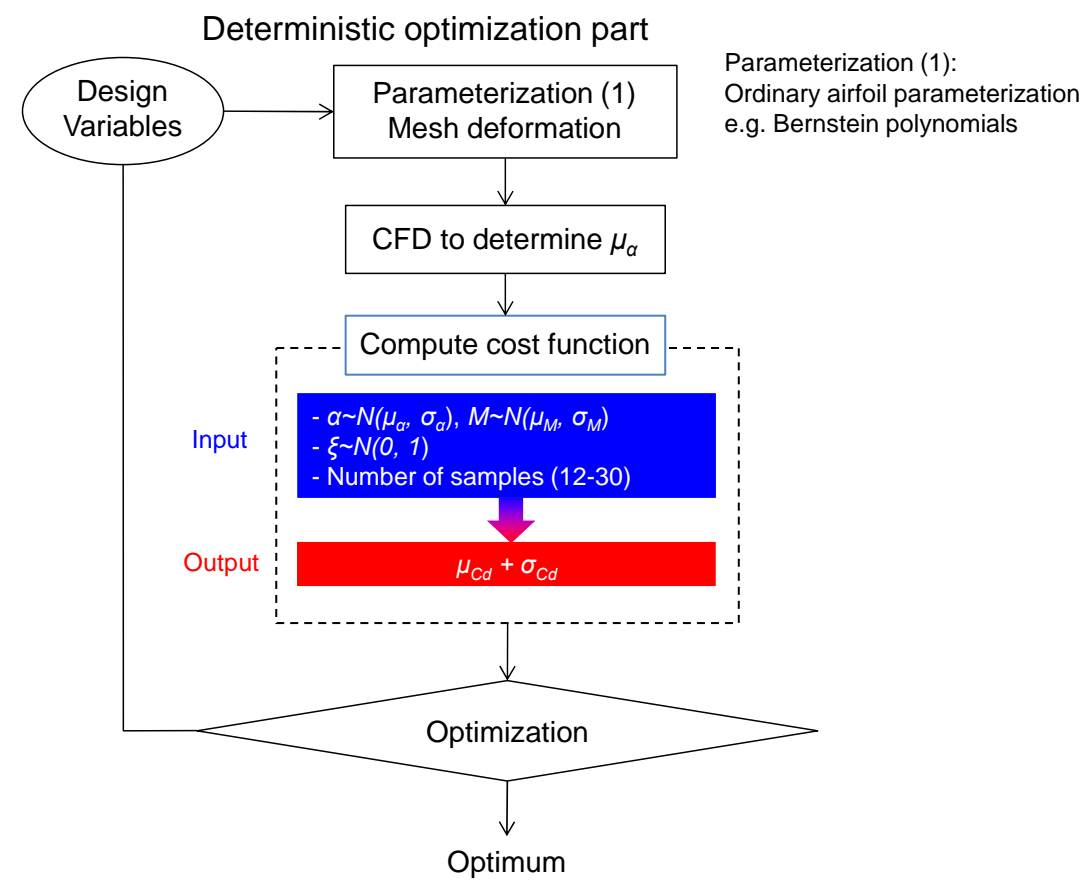

Figure 4: Overview of robust design optimization framework (deterministic optimization part).

Next, to construct a surrogate model of $C_{d}$, CFD calculations with the TAU-code are conducted on the samples. When a gradient-enhanced surrogate model is considered, additional adjoint calculations are run with the TAU-code to obtain gradients of $C_{d}$ with respect to the uncertainty parameters $\mathbf{a}$ and $\boldsymbol{\xi}$. The computation of the samples values and their gradients is obviously the most time-consuming part of the entire process. Therefore, the number of DOE samples has the largest impact on the framework in terms of efficiency. Note, however, that each sample is independent of all other samples. Thus, all samples can be computed in parallel.

Finally, a surrogate model is constructed by using Kriging or GEK. Then the mean, $\mu$, and standard deviation, $\sigma$, of the drag coefficient are calculated based on the surrogate model by direct MC quadrature as:

$$
\left\{\begin{array}{l}
\mu_{C d}(\chi, \mathbf{a}, \boldsymbol{\xi})=\frac{1}{N} \sum_{i=1}^{N}\left(C_{d}(\chi, \mathbf{a}, \boldsymbol{\xi})\right) \\
\sigma_{C_{d}}(\chi, \mathbf{a}, \boldsymbol{\xi})=\sqrt{\frac{1}{N-1} \sum_{i=1}^{N}\left(C_{d}(\chi, \mathbf{a}, \boldsymbol{\xi})-\mu_{C_{d}}(\chi, \mathbf{a}, \boldsymbol{\xi})\right)^{2}}
\end{array}\right.
$$

where $N$ is a sufficiently large number of samples $\left(N=10^{6}\right.$ in this paper). As described in Section 2.3, the error to the true value on a surrogate model is negligible when the number of samples is large. Therefore the quality of the surrogate models is the most important factor in terms of accuracy of the statistics.

In the next section, both Kriging and GEK are investigated in this respect by varying the number of samples used to construct the surrogate models. 


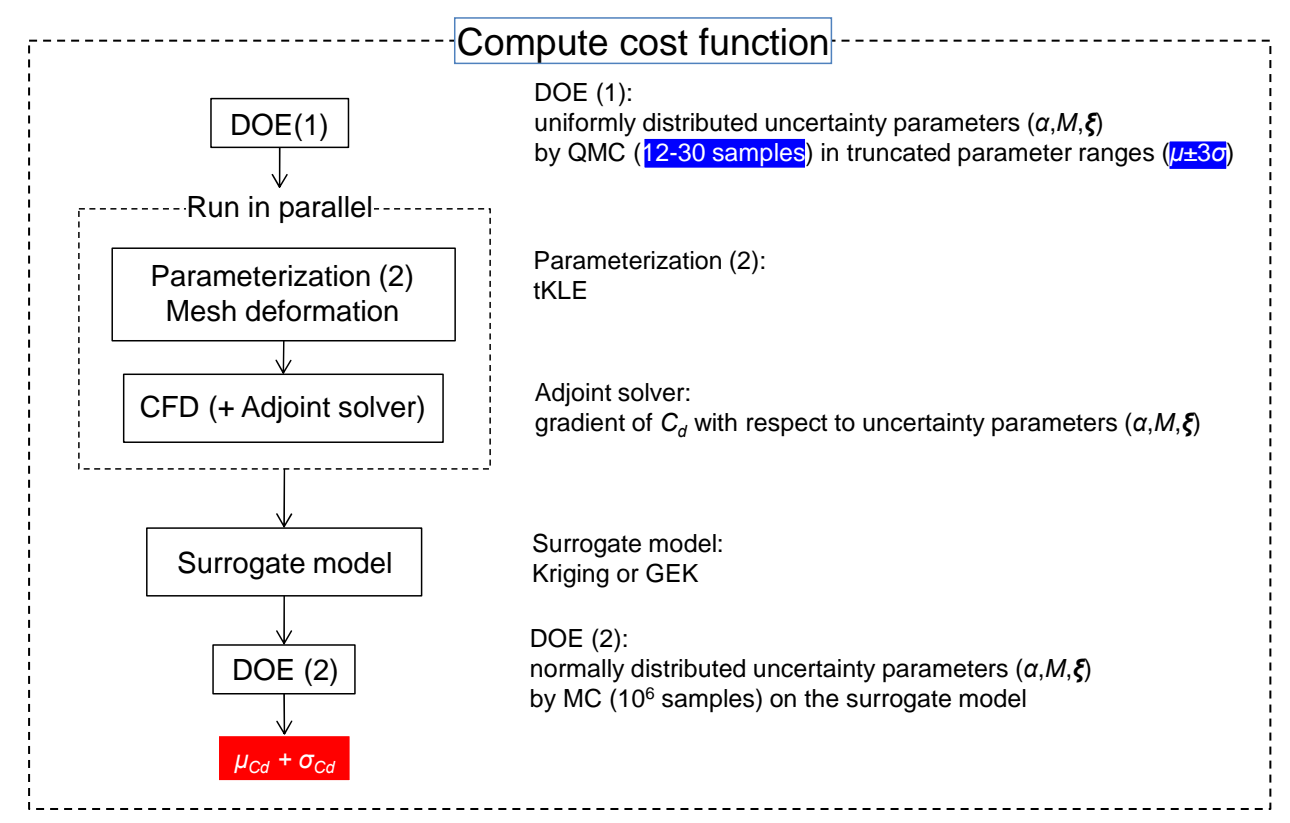

Figure 5: Overview of robust design optimization framework (evaluation of stochastic cost function).

\section{RESULTS AND DISCUSSION}

\subsection{Accuracy of cost function}

In this section, the cost function $f$, which is defined by Eq. (2), calculated for different surrogate models is compared with the true cost function $\hat{f}$ for the baseline airfoil configuration The true cost function $\hat{f}$, which acts as a reference, was calculated according to Eq. (3) by the direct QMC quadrature based on $10^{5}$ samples computed with TAU for RAE2822 as the given nominal airfoil shape. Note that in general in RDO, the true cost function $\hat{f}$ for a designed nominal airfoil shape at each optimization cycle is unknown.

As mentioned in the previous sections, the accuracy of the statistics at every optimization cycle depends on that of the surrogate models. As mentioned in Section 2.3, the cost function $f$ is almost uniquely determined once a surrogate model is constructed by Eq. (3). As for the construction of a surrogate model, once the sample points are fixed, the Kriging-based surrogate model is uniquely determined by its Gaussian correlation function and the likelihood function. When constructing a Kriging model, model parameters, so-called hyper-parameters, need to be tuned based on the sampled data using MLE, which is obtained in this study by the differential evolution as a global optimization method.

In our RDO framework, a surrogate model is constructed at every optimization cycle to obtain the cost function $f$. The surrogate model needs to be accurately constructed many times to obtain the cost function $f$, which is unknown but should be robustly evaluated to sample points $\hat{f}$ for a better guide to the next steps in whole the optimization cycles.

Therefore as a first step of estimation of the cost function $f$, we focus here on the initial guess, that is, the distribution of sample points. In practice, different 100 sets of distributions of sample points are derived from the Sobol sequence. Then, the dependency of accuracy of $f$ (in surrogate models) on the sample points are investigated by using two kinds of the num- 
ber of sample points: 12 and 30, and by using two types of surrogate models: Kriging and GEK. Therefore, we investigated the following four approaches: (a) 12 samples with Kriging, (b) 30 samples with Kriging, (c) 12 samples with GEK, (d) 30 samples with GEK.

As defined in the previous section, the number of uncertainties is fixed to 12 . Therefore, $12 \times 12$ or $30 \times 12$ sequences are generated to construct one surrogate model. These different sets of sequences are extracted 100 times from the Sobol sequences. Then 100 sets of differently distributed sample points are generated. The mean $\mu_{f}$ and standard deviation $\sigma_{f}$ of these 100 cost functions $f_{1} \sim f_{100}$ in the 100 surrogate models are calculated to investigate how accurate the approach to construct surrogate models is by comparison with the true cost function $\hat{f}$.

Figure 6(i) shows distributions of the 100 predicted cost functions $f_{1} \sim f_{100}$ obtained by 100 different sets of sample points by the four approaches (a) (b). The stochastic values of these cost functions $f_{1} \sim f_{100}$ are then calculated. The standard deviation $\sigma_{f}$ and mean $\mu_{f}$ of $f_{1} \sim f_{100}$ is summarized in Fig. 6(ii). The mean values $\mu_{f}$ are depicted by adjusting to the relative error to the true cost function $\hat{f}$ as $\left\|\left(\mu_{f}-\hat{f}\right) / \hat{f}\right\|$. In this figure a correlation can be observed between $\sigma_{f}$ and $\left\|\left(\mu_{f}-\hat{f}\right) / \hat{f}\right\|$, those of which express the accuracy in terms of the distribution of sample points. The result using the strategy (d) 30 samples with GEK, can provide the mean of cost function $\mu_{f}$ with only $0.0003 \%$ relative error to the true value $\hat{f}$ and with 0.9 count (here one count $=10^{-4}$ ) for the standard deviation $\sigma_{f}$ as the error bound. Further improvement of efficiency by using less sample points can be expected by applying adaptive sampling strategies, which will be the next step of estimation of the cost function $f$.

Finally, the averaged computational costs to construct a surrogate model on one core on a cluster are summarized in Table 1 . They are proportional to between the square and the cube of the samples points for both Kriging and GEK. For instance, the GEK using 30 samples costs around 10 times more than that using 12 samples. However, they are all quite small compared with one general CFD computational time. In this study, we fixed the optimizer for the MLE to a global one. We note that it was pointed out in [20] that global optimizers can provide a more accurate surrogate model in terms of not only the MLE but also the sample points.

\subsection{Application to robust design optimization (RDO)}

The developed RDO framework was applied to the transonic RAE2822 airfoil, which is one of the URMIDA test cases, by taking into account an uncertain angle of attack and Mach number, and a large number of geometrical uncertainties. Based on the results in the previous section, the GEK surrogate model used to evaluate the robust objective function at each optimization cycle is constructed by computing 30 samples and the corresponding partial gradients with TAU and its adjoint flow solver. All the other settings are described in Section 3. Figure 7 shows the history of the cost function $f$ defined in Eq. (2) and the nominal design variables $\chi$. We can observe that there is a relatively large amplitude of oscillations of the cost function $f$ after 350 optimization cycles, while the design variables $\chi$ do not vary any more. However, this amplitude is the smallest in the four types of surrogate models mentioned 
(i)

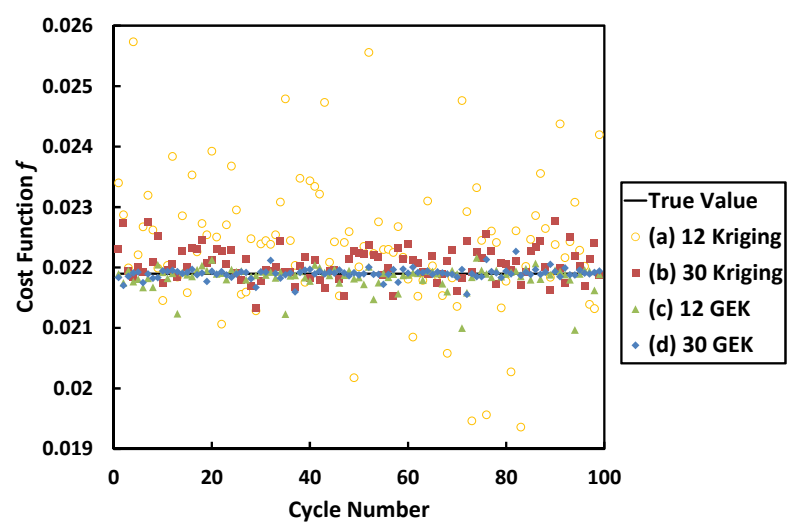

(ii) $\times$

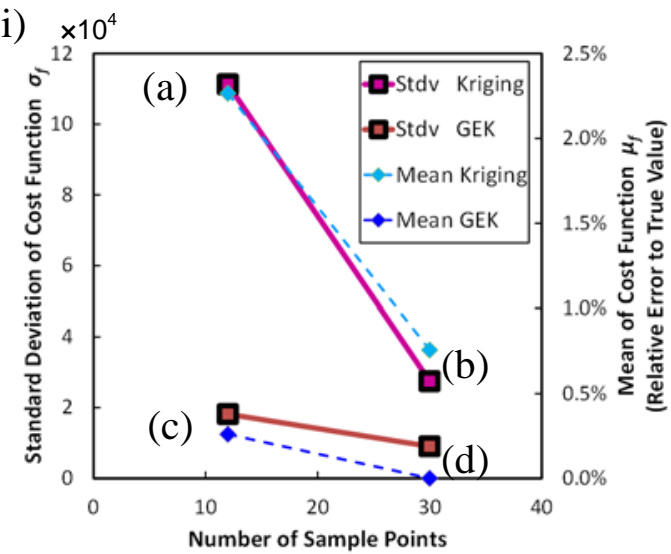

Figure 6: Dependency of accuracy of cost function on number of sample points and surrogate methods used to construct a surrogate model (left), and comparison of mean and standard deviation of the cost function based on (a) 12 samples with Kriging, (b) 30 samples with Kriging, (c) 12 samples with GEK, (d) 30 samples with GEK (right).

\begin{tabular}{lll}
\hline & 12 samples & 30 samples \\
\hline Kriging & (a) $0.4 \mathrm{sec}$. & (b) $0.4 \mathrm{sec}$ \\
GEK & (c) $1.7 \mathrm{sec}$. & (d) $14.6 \mathrm{sec}$ \\
\hline
\end{tabular}

Table 1: Averaged computational costs for construction of a surrogate model on one core on a cluster.

in the previous sub-section. Figure 8 depicts a comparison of configurations of the initial airfoil (RAE2822) the robust airfoil. A designed airfoil configuration by the deterministic design optimization (DDO) is also shown in this figure. Table 2 shows $\mu_{C d}$ and $\sigma_{C d}$ of the initial, robust, and the deterministic designed airfoils. These stochastic values $\mu_{C d}$ and $\sigma_{C d}$ were reevaluated by using the same strategy as that in Section 4.1. They are calculated by taking the average of 100 stochastic cost functions $f$ predicted by 30 sample points and the GEK surrogate model. It can be observed that both $\mu_{C d}$ and $\sigma_{C d}$ were successfully decreased. The total iteration number of the Supblex-based RDO for 10 design variables was 564. All computations were performed on a single core apart from the CFD computations which were ran in parallel on 24 cores. In terms of computational time, the total wall clock time was about 160 hours. The efficiency of the total RDO optimization could be improved by a two-step optimization strategy. For instance, at first deterministic design optimization (DDO) is conducted to the initial configuration and then a local optimizer such as gradient-based optimization algorithm is applied to the deterministic designed airfoil for RDO.

In this paper, we focused on accuracy and efficiency of construction of surrogate models in terms of stochastic values such as mean and standard deviation. The developed framework can also be applied for investigating the Pareto front in this robust optimization setting. Moreover, this surrogate-assisted cost function evaluation can also be applied to reliabilitybased design optimization by considering the probability density function of $C_{d}$.

As can be confirmed by Fig. 6 in the previous sub-section, there is a correlation between the amplitude of the oscillations of the cost function and the accuracy of the surrogate models. 
The amplitude of the oscillations can be further reduced by improving the accuracy of the underlying surrogate models, e.g., by employing adaptive sampling strategies.

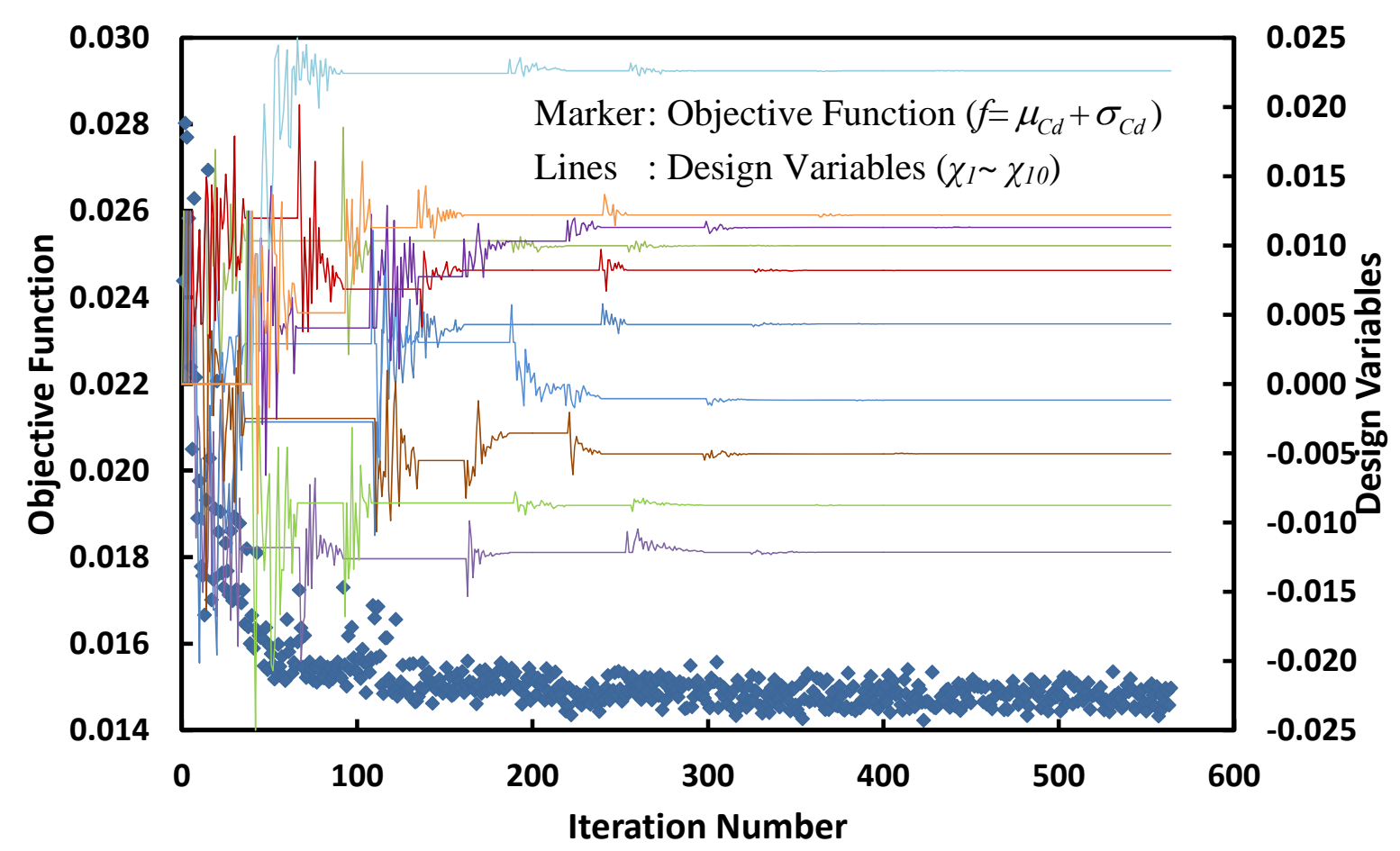

Figure 7: History of objective function and design variables in robust design optimization (RDO).

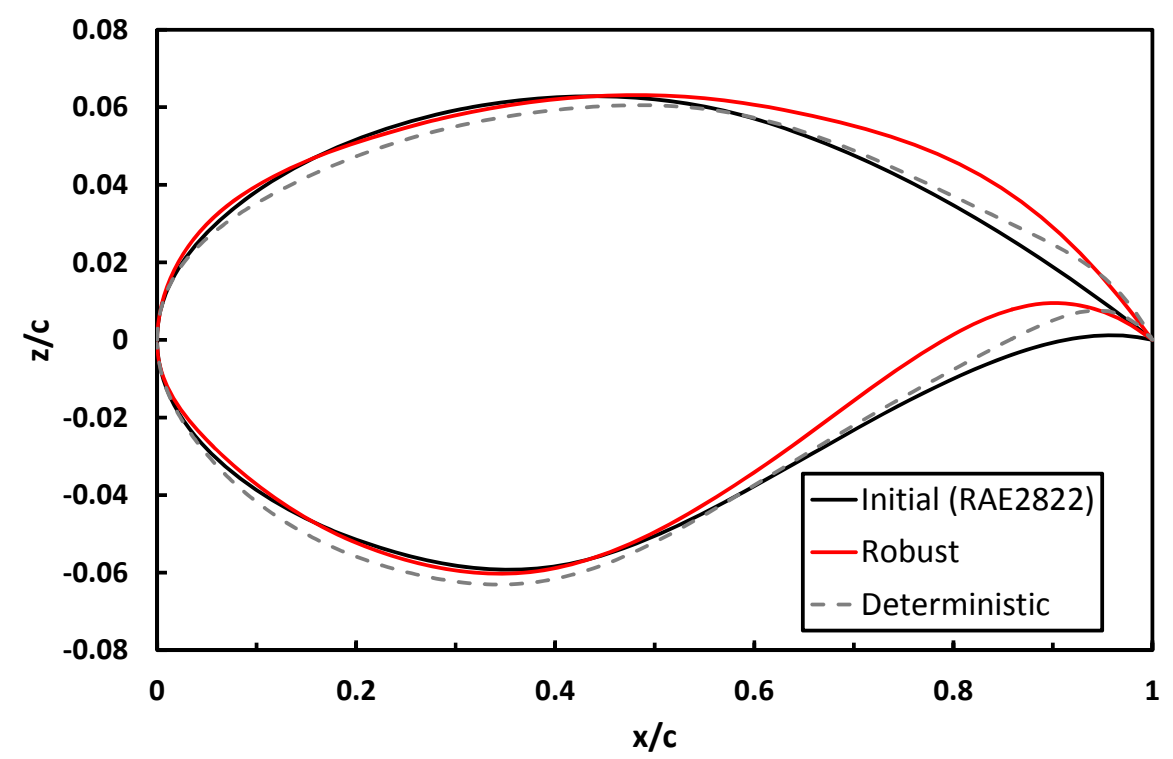

Figure 8: Comparison of initial (RAE2822), robust and deterministic designed airfoil geometries. 


\begin{tabular}{cccc}
\hline & Initial & Robust & Deterministic \\
\hline$\mu_{C d}$ & 192.4 cts. & 137.9 cts. & 134.2 cts. \\
$\sigma_{C d}$ & 26.6 cts. & 8.8 cts. & 12.6 cts. \\
\hline
\end{tabular}

Table 2: Mean and standard deviation of $C_{d}$ of initial, robust, and deterministic designed airfoils (1cts. $=10^{-4}$ ).

\section{CONCLUSIONS}

A robust optimization framework for the aerodynamic design of airfoils was developed. To efficiently calculate the stochastic values which are used to evaluate the robustness measure in every optimization cycle, we employ design of experiments methods and Kriging-based surrogate models. A large number of geometrical uncertainties was modeled using a KarhunenLoève expansion. The stochastic output values due to two operational uncertainties and 10 geometrical uncertainties were evaluated quite accurately based on at most 30 CFD computations and corresponding adjoint computations, the latter providing gradient information with respect to the 12 uncertainty parameters. These stochastic methods were integrated into an inhouse framework for deterministic shape optimization. The developed framework was used to perform robust optimization of a transonic RAE2822 airfoil, which is one of the test cases in the EU project UMRIDA. The stochastic values such as the mean and standard deviation of the drag coefficient were successfully minimized and a new, more robust nominal configuration was proposed by this efficient robust design optimization framework.

\section{ACKNOWLEDGEMENTS}

Part of the research leading to this work was supported by the UMRIDA (Uncertainty Management for Robust Industrial Design in Aeronautics) project, funded by the European Commission under grant number ACP3-GA-2013-605036, and by the Digital-X project at DLR. The authors would like to thank their colleagues Niklas Karcher, Thomas Franz, and Dr. Matteo Ripepi for discussions and help with DLR's SMARTy toolbox and Caslav Ilic and Mohammad Abu-Zurayk for help with DLR's Pyranha toolbox.

\section{REFERENCES}

[1] UMRIDA official website: http://www.umrida.eu.

[2] R. Duvigneau, Aerodynamic Shape Optimization with Uncertain Operating Conditions using Metamodels. Research Report 6143, INRIA, March, 2007.

[3] M. Martinelli, R. Duvigneau, On the use of second-order derivatives and metamodelbased Monte-Carlo for uncertainty estimation in aerodynamics. Computers \& Fluids, Volume 39, Issue 6, 953-964, 2010.

[4] M. Dodson, T.P. Geoffrey, Robust Aerodynamic Design Optimization Using Polynomial Chaos. Journal of Aircraft, Vol. 46, No. 2, 635-646, 2009. 
[5] C. Schillings, V. Schulz, On the influence of robustness measures on shape optimization with stochastic uncertainties. Optimization and Engineering, Volume 16, Issue 2, 347386, 2014.

[6] D. Liu, Efficient Quantification of Aerodynamic Uncertainties Using GradientEmploying Surrogate methods. Notes on Numerical Fluid Mechanics and Multidisciplinary Design, Volume 122, Management and Minimisation of Uncertainties and Errors in Numerical Aerodynamics, 283-296, 2013.

[7] D. Liu, S. Görtz, Influence of reduced-order modelling of geometrical uncertainties on statistics. 7. Dresdner-Probabilistik-Workshop, Dresden, October, 2014.

[8] M. Galle, T. Gerhold, J. Evans, Parallel computation of turbulent flows around complex geometries on hybrid grids with the DLR-TAU code, in: A. Ecer, D.R. Emerson (Eds.), Proc. 11th Parallel CFD Conf., Williamsburg, VA, North-Holland, May 23-26, 1999.

[9] T. Gerhold, V. Hannemann, D. Schwamborn, On the validation of the DLR-TAU code, in: W. Nitsche, H.-J. Heinemann, R. Hilbig (Eds.), New Results in Numerical and Experimental Fluid Mechanics, in: Notes on Numerical Fluid Mechanics, vol. 72, Vieweg, ISBN 3-528-03122-0, 1999, pp. 426-433.

[10] D. Schwamborn, T. Gerhold, R. Heinrich, The DLR TAU-code: Recent applications in research and industry, invited lecture, in: P. Wesseling, E. Oate, J. Priaux (Eds.), Proceedings of the European Conference on Computational Fluid Dynamics (ECCOMAS CFD 2006), The Netherlands, 2006.

[11] S.R. Allmaras, F.T. Johnson, P.R. Spalart, Modifications and Clarifications for the Implementation of the Spalart-Allmaras Turbulence Model. Seventh International Conference on Computational Fluid Dynamics (ICCFD7), ICCFD7-1902, Hawaii, July, 2012.

[12] R. Heinrich, K. Reimer, A. Michler, Multidisciplinary simulation of maneuvering aircraft interacting with atmospheric effects using the DLR TAU code. RTO AVT-189 Specialists' Meeting on Assessment of Stability and Control Prediction Methods for Air and Sea Vehicles, Portsdown West, October 12-14, 2011.

[13] I.M. Sobol, Distribution of points in a cube and approximate evaluation of integrals. $Z h$. Vychisl. Mat. Mat. Fiz., Volume 7, Number 4, 784-802, 1967.

[14] S. Joe, F. Y. Kuo, Remark on Algorithm 659: Implementing Sobol's quasirandom sequence generator, ACM Trans. Math. Softw. 29, 49-57, 2003

[15] S. Joe, F. Y. Kuo, Constructing Sobol sequences with better two-dimensional projections, SIAM J. Sci. Comput. 30, 2635-2654, 2008.

[16] Z.H. Han, S. Görtz, R. Zimmermann, Improving Variable-Fidelity Surrogate modeling via Gradient-Enhanced Kriging and a Generalized hybrid Bridge Function. Journal of Aerospace Science and Technology, Vol. 25, Issue 1, 2013.

[17] R. Dwight, J. Brezillon, Effect of approximations of the discrete adjoint on gradientbased optimization, AIAA Journal, Vol. 44, Issue 12, 3022-3071, 2006.

[18] J. Brezillon, M. Abu-Zurayk, Aerodynamic Inverse Design Framework Using Discrete Adjoint Method, Notes on Numerical Fluid Mechanics and Multidisciplinary Design, 121. Springer Verlag. pp. 489-496. January 2013.

[19] T. Rowan, Functional Stability Analysis of Numerical Algorithms, Ph.D. thesis, Department of Computer Sciences, University of Texas at Austin, 1990. 
[20] L. Zhao, K.K. Choi, I. Lee, A Metamodel Method Using Dynamic Kriging and Sequential Sampling. AIAA-2010-9391, Fort Worth, September, 2010. 